\title{
On matrix type corings, algebra coverings and Čech cohomology
}

\author{
Andrzej Sitarz *† \\ Institute of Physics, Jagiellonian University, \\ Reymonta 4, 30-059 Kraków, Poland
}

\begin{abstract}
We investigate the a matrix-type coring associated to a complete covering of an algebra, its Amitsur complex and propose a definition for the related Čech cohomology relative to the covering.
\end{abstract}

\section{Introduction}

The definition and examples of algebra coverings, as given by Calow and Matthes [1] provided a nice set of examples of canonical corings [3]. In this note, we elaborate further on the construction and define a version of Čech cohomology respective to the covering using the cochain complexes based on Amitsur complex.

\subsection{Preliminaries}

We recall here the notation and definition. Let $A$ be a unital algebra and $I_{i} \subset A, i=1,2, \ldots N$, be two-sided ideals in $A$. We call $A_{j}=A / I_{j}$ and $A_{i j}=A /\left(I_{i}+I_{j}\right)$, further $B=\bigoplus_{i} A_{i}$ and $B^{\prime}=\bigoplus_{i, j} A_{i j}$.

*The author acknowledges the Alexander von Humboldt Fellowship at the Mathematisches Institut der Heinrich-Heine-Universität, Universitätsstrasse 1, 40225 Düsseldorf, Germany

†Partially supported by Polish Government grants 115/E-343/SPB/6.PR UE/DIE 50/2005-2008 and 189/6.PRUE/2007/7 
Definition 1.1 (Definition 2,[1]). The ideals $\left\{I_{i}\right\}_{i \in \mathcal{I}}$ are called a covering of $A$ if their intersection is $\{0\}$. We call the covering complete if the following sequence of $A$-bimodules is exact:

$$
0 \longrightarrow A \stackrel{\pi}{\longrightarrow} B \stackrel{\tau}{\longrightarrow} B^{\prime}
$$

where

$$
\pi=\bigoplus_{i} \pi_{i}, \pi_{i}: A \rightarrow A_{i}
$$

and

$$
\tau: \bigoplus_{i} A_{i} \rightarrow \bigoplus_{i, j} A_{i j}: \tau\left(\bigoplus_{i} x_{i}\right)=\oplus_{i, j}\left(\pi_{i j}^{i}\left(x_{i}\right)-\pi_{i j}^{j}\left(x_{j}\right),\right),
$$

where $\pi_{i j}^{i}: A / I_{i} \rightarrow A /\left(I_{i}+I_{j}\right)$.

Definition 1.2. The coring $\mathcal{C}\left(A,\{I\}_{\mathcal{I}}\right)$ associated to the covering $A,\left\{I_{i}\right\}_{i \in \mathcal{I}}$ is the Sweedler coring of the algebra extension $A \hookrightarrow B$.

Remark 1. The coring $\mathcal{C}$ is a generalization of a matrix coring, where the $(i, j)$-matrix entry takes values in $A_{i} \otimes_{A} A_{j}$. The latter can be, for unital algebras identified (as $A$-bimodule) with $A_{i j}$.

If we denote $e_{i j}=\pi^{i}\left(1_{A}\right) \otimes_{A} \pi^{j}\left(1_{A}\right)$ then for the coproduct and counit in $\mathcal{C}$ we have:

$$
\begin{aligned}
\Delta e_{i j} & =\pi^{i}\left(1_{A}\right) \otimes_{B}\left(\sum_{k} \pi^{k}\left(1_{A}\right)\right) \otimes_{A} \pi_{j}\left(1_{A}\right) \\
& =\sum_{k}\left(\pi^{i}\left(1_{A}\right) \otimes_{A} \pi^{k}\left(1_{A}\right)\right) \otimes_{B}\left(\pi^{k}\left(1_{A}\right) \otimes_{A} \pi^{j}\left(1_{A}\right)\right) \\
& =\sum_{k} e_{i k} \otimes_{B} e_{k j} . \\
\varepsilon\left(e_{i j}\right) & =\delta_{i j} \pi_{i}\left(1_{A}\right) .
\end{aligned}
$$

Note that although formally the coproduct formula is the same as in the case of a matrix coring, only in the case $A_{i} \equiv A, i \in I$, we recover the usual definition of the matrix coring.

We have:

Theorem $1.3([3], 3.4)$. The coring $\mathcal{C}$ is Galois if the covering is complete. 


\section{The Čech cohomology of noncommutative coverings}

In the classical situation of coverings of a topological space with open sets, with some additional data, we can easily introduce the notion of Čech cochain complex relative to the covering.

We show that in the noncommutative situation, this is also possible and we relate the construction to the Amitsur complex of the coring related to the covering. However, for the purpose of the construction we need to adapt the notion of ringed space to the algebra case and define the corresponding complex.

\section{1 Čech complex of rings}

Let $\mathcal{I}$ be a finite set of indices and $P(\mathcal{I})$ be the set of its ordered subsets. We say that for any two ordered subsets $\zeta, \vartheta$ of $\mathcal{I}, \zeta \subset \vartheta$ if $\zeta$ is a subset of $\vartheta$ and the order in $\zeta$ is inherited from the order in $\vartheta$. We shall use $|\zeta|$ to denote cardinality of the subset $\zeta$.

We assume that $\mathcal{R}$ is a functor from $P(\mathcal{I})$ to the category of unital rings. That is, for any $\vartheta \in P(\mathcal{I})$ there is a unital ring $\mathcal{R}(\vartheta)$, and for any $\zeta, \vartheta \in P(\mathcal{I})$ such that $\zeta \subset \vartheta$ there is a ring homomorphism:

$$
r_{\vartheta}^{\zeta}: R(\zeta) \rightarrow R(\vartheta)
$$

We assume that $r_{\zeta}^{\zeta}$ is always an identity morphism, and that for any three $\zeta \subset \eta \subset \vartheta \subset P(\mathcal{I})$ we have:

$$
r_{\vartheta}^{\eta} \circ r_{\zeta}^{\eta}=r_{\zeta}^{\eta}
$$

We shall identify $R=\mathcal{R}(\emptyset)$.

Since $\mathcal{R}(\emptyset) \hookrightarrow \bigoplus_{i} \mathcal{R}(\{i\})$, is an embedding, we have a similar situation of a ring extension as before and we can associate a Sweedler coring with the construction, which we shall call $\mathcal{C}(\mathcal{I})$.

\subsection{The Amitsur complex of a coring}

We recall here the definition of the Amitsur complex for the canonical Sweedler coring $\mathcal{C}=B \otimes_{A} B$ [2]. We use similar notation as before, here $A_{\mathcal{R}}=\mathcal{R}(\emptyset)$ and $B_{\mathcal{R}}=\bigoplus_{i} \mathcal{R}(\{i\})$. 
Definition 2.1. Let $\left\{C^{n}\right\}_{n=0, \ldots}$ be the following complex:

$$
C^{0}=B_{\mathcal{I}}, \quad C^{n}=B_{\mathcal{I}}^{\otimes_{A_{\mathcal{R}}}(n-1)} \cong \mathcal{C}^{\otimes_{B_{\mathcal{R}}}}, n \geq 1
$$

with the map:

$$
\begin{aligned}
& d(x)=1 \otimes_{A_{\mathcal{R}}} x-x \otimes_{A_{\mathcal{R}}} 1, \quad x \in C^{0}, \\
& d(\omega)=1 \otimes_{A_{\mathcal{R}}} \omega-b^{\prime}(\omega)+(-1)^{n+1} \omega \otimes_{A_{\mathcal{R}}} 1, \quad \omega \in C^{n}, n \geq 1,
\end{aligned}
$$

where $b^{\prime}$ is the coboundary of the Hochschild bar complex constructed with the coring coproduct:

$$
b^{\prime}\left(c_{0} \otimes_{B_{\mathcal{R}}} c_{1} \otimes_{B_{\mathcal{R}}} \cdots \otimes_{B_{\mathcal{R}}} c_{n}\right)=\sum_{i=0}^{n}(-1)^{n} c_{0} \otimes_{B_{\mathcal{R}}} c_{1} \otimes_{B_{\mathcal{R}}} \cdots \Delta c_{i} \cdots \otimes_{B_{\mathcal{R}}} c_{n} .
$$

which, for the Sweedler corings is expressed using the equivalent presentation as:

$$
\begin{array}{r}
b^{\prime}\left(x_{0} \otimes_{A_{\mathcal{R}}} x_{1} \otimes_{A_{\mathcal{R}}} \cdots \otimes_{A_{\mathcal{R}}} x_{n+1}\right)= \\
\sum_{i=0}^{n}(-1)^{n} x_{0} \otimes_{A_{\mathcal{R}}} x_{1} \otimes_{A_{\mathcal{R}}} \cdots x_{i} \otimes_{A_{\mathcal{R}}} 1 \otimes_{A_{\mathcal{R}}} x_{i+1} \cdots \otimes_{A_{\mathcal{R}}} x_{n+1} .
\end{array}
$$

We have (see [2] 29.5)

Proposition 2.2. The Amitsur complex of a Galois coring is acyclic. Hence the Amitsur complex of a coring related to a complete covering is acyclic.

Therefore, to obtain more information, which arises from the covering we need to construct a different cochain complex, associated to the functor $\mathcal{R}$.

Definition 2.3. Let $\phi$ be a map between the complex of the coring of covering $\mathcal{C}(\mathcal{R})$ and the $\mathbb{Z}$-graded module $\bigoplus_{n \in \mathbb{Z}} S^{n}$, where

$$
S^{n}=\bigoplus_{\zeta \in P(\mathcal{I}),|\zeta|=n} \mathcal{R}(\zeta)
$$

defined as:

$$
\phi\left(y_{i_{1}} \otimes_{B_{\mathcal{R}}} y_{i_{2}} \cdots \otimes_{B_{\mathcal{R}}} y_{i_{n}}\right)=r_{\zeta}^{i_{1}}\left(\Phi_{I_{1}}\left(y_{i_{1}}\right)\right) r_{\zeta}^{i_{2}}\left(\Phi_{I_{2}}\left(y_{i_{2}}\right)\right) \cdots r_{\zeta}^{i_{n}}\left(\Phi_{I_{n}}\left(y_{i_{n}}\right)\right),
$$


for any $\zeta=\left\{i_{1}, i_{2}, \ldots, i_{n}\right\} \in P(I), y_{i_{k}} \in A_{i_{k}}$, and:

$$
\phi\left(y_{i_{1}} \otimes_{B_{\mathcal{R}}} y_{i_{2}} \cdots \otimes_{B_{\mathcal{R}}} y_{i_{n}}\right) \equiv 0,
$$

for any $\left\{i_{1}, i_{2}, \ldots, i_{n}\right\}$ which are not in $P(\mathcal{I})^{1}$. The maps $\Phi_{i_{k}}$ denote some chosen ring homomorphisms between $A_{i_{k}}$ and $\mathcal{R}\left(\left\{i_{k}\right\}\right)$, in fact, we have $\phi\left(y_{i}\right) \equiv \Phi_{i}\left(y_{i}\right)$.

Proposition 2.4. $S^{n}$ is a cochain complex, with the coboundary: map $d^{\prime}$ : $S^{n} \rightarrow S^{n+1}$ :

$$
d^{\prime} x_{\zeta}=\sum_{\eta, \zeta \subset \eta,|\eta|=|\zeta|+1}(-1)^{|\eta / \zeta|} r_{\eta}^{\zeta}\left(x_{\zeta}\right),
$$

where $|\eta / \zeta|$ denotes position on which the difference between $\eta$ and $\zeta$ occurs. Then $\phi$ is a morphism of cochain complexes.

We call the cochain complex $S(\mathcal{R})=\left\{\left(S^{n}, d^{\prime}\right)\right\}_{n}$ the Čech complex associated to functor $\mathcal{R}$.

Proof. First of all, let us see that the map $\phi$ is well-defined (the definition of $\phi$ is set for tensor products and we need to check that it remains correct for tensor products over $B_{\mathcal{R}}$.) We have:

$$
\begin{aligned}
\phi( & \left.\cdots y^{i_{k}} \pi_{i_{k}}(y) \otimes_{B_{\mathcal{R}}} y^{i_{k+1}} \cdots\right)=\cdots r_{\zeta}^{i_{k}}\left(\Phi_{i_{k}}\left(y_{i_{k}} \pi_{i_{k}}(y)\right)\right) r_{\zeta}^{i_{k+1}}\left(\Phi_{i_{k+1}}\left(y_{i_{k+1}}\right)\right) \cdots \\
& =\cdots r_{\zeta}^{i_{k}}\left(\Phi_{i_{k}}\left(y_{i_{k}}\right) \Phi_{i_{k}}\left(\pi_{i_{k}}(y)\right)\right) r_{\zeta}^{i_{k+1}}\left(\Phi_{i_{k+1}}\left(y_{i_{k+1}}\right)\right) \cdots \\
& =\cdots r_{\zeta}^{i_{k}}\left(\Phi_{i_{k}}\left(y_{i_{k}}\right)\right) r_{\zeta}^{i_{k}}\left(\Phi_{i_{k}}\left(\pi_{i_{k}}(y)\right)\right) r_{\zeta}^{i_{k+1}}\left(\Phi_{i_{k+1}}\left(y_{i_{k+1}}\right)\right) \cdots \\
& =\cdots r_{\zeta}^{i_{k}}\left(\Phi_{i_{k}}\left(y_{i_{k}}\right)\right) r_{\zeta}^{i_{k+1}}\left(\Phi_{i_{k+1}}\left(\pi_{i_{k+1}}(y)\right)\right) r_{\zeta}^{i_{k+1}}\left(\Phi_{i_{k+1}}\left(y_{i_{k+1}}\right)\right) \cdots \\
& =\cdots r_{\zeta}^{i_{k}}\left(\Phi_{i_{k}}\left(y_{i_{k}}\right)\right) r_{\zeta}^{i_{k+1}}\left(\Phi_{i_{k+1}}\left(\pi_{i_{k+1}}(y) y_{i_{k+1}}\right)\right) \cdots \\
& =\phi\left(\cdots y^{i_{k}} \otimes_{B_{\mathcal{R}}} r_{i_{k+1}}(y) y^{i_{k+1}} \cdots\right) .
\end{aligned}
$$

\footnotetext{
${ }^{1}$ This happens when at least two of the indices are identical.
} 
As the next step, we check that the map $d^{\prime}$ is a coboundary:

$$
\begin{aligned}
\left(d^{\prime}\right)^{2} x_{\zeta} & =d^{\prime}\left(\sum_{\eta, \zeta \subset \eta,|\eta|=|\zeta|+1}(-1)^{|\eta / \zeta|} r_{\eta}^{\zeta}\left(x_{\zeta}\right)\right) \\
& =\sum_{\vartheta, \vartheta \subset \zeta,|\zeta|=|\vartheta|+1} \sum_{\eta, \zeta \subset \eta,|\eta|=|\zeta|+1}(-1)^{|\vartheta / \zeta|}(-1)^{|\eta / \zeta|} r_{\vartheta}^{\eta} \circ r_{\eta}^{\zeta}\left(x_{\zeta}\right) \\
& =\sum_{\vartheta, \vartheta \subset \zeta,|\zeta|=|\vartheta|+2}\left((-1)^{\left|\eta_{1} / \zeta\right|+\left|\vartheta / \eta_{1}\right|}+(-1)^{\left|\eta_{2} / \zeta\right|+\left|\vartheta / \eta_{2}\right|}\right) r_{\vartheta}^{\zeta}\left(x_{\zeta}\right) \\
& =0,
\end{aligned}
$$

where we have used (2.1) and the fact that for each $\vartheta \subset \zeta$ such that $|\vartheta|+2=$ $|\zeta|$ there are two possibilities for $\vartheta \subset \eta_{1,2} \subset \zeta$ with $|\eta|=|\vartheta|+1$, which we denoted $\eta_{1}, \eta_{2}$. It is easy to verify that:

$$
(-1)^{\left|\eta_{1} / \zeta\right|+\left|\vartheta / \eta_{1}\right|}+(-1)^{\left|\eta_{2} / \zeta\right|+\left|\vartheta / \eta_{2}\right|}=0
$$

which ends the verification.

Finally, to verify that $\phi$ is a cochain complex morphism we calculate now using $x_{i}=\phi\left(y_{i}\right)$. First:

$$
\begin{aligned}
d^{\prime} \circ \phi & \left(x_{i_{0}} \otimes_{R} x_{i_{1}} \cdots \otimes_{R} x_{i_{n}}\right)=d^{\prime}\left(r_{\zeta}^{i_{0}}\left(x_{i_{0}}\right) r_{\zeta}^{i_{1}}\left(x_{i_{1}}\right) \cdots r_{\zeta}^{i_{n}}\left(x_{i_{n}}\right)\right) \\
= & \sum_{i \in I / \zeta} \sum_{k=0}^{n+1}(-1)^{k} r_{\zeta}^{\eta(k)}\left(r_{\zeta}^{i_{0}}\left(x_{i_{1}}\right) r_{\zeta}^{i_{1}}\left(x_{i_{1}}\right) \cdots r_{\zeta}^{i_{n}}\left(x_{i_{n}}\right)\right) \\
= & \sum_{i \in I / \zeta} \sum_{k=0}^{n+1}(-1)^{k} r_{\eta(k)}^{i_{0}}\left(x_{i_{0}}\right) r_{\eta(k)}^{i_{1}}\left(x_{i_{1}}\right) \cdots r_{\eta(k)}^{i_{n}}\left(x_{i_{n}}\right)
\end{aligned}
$$

where $\eta(k)$ denotes the ordered subset of $P(I)$, containing $\zeta$ and $i \notin \zeta$ on $k+1$-th place. 
On the other hand, calculating $\phi \circ d$,

$$
\begin{aligned}
\phi \circ d & \left(x_{i_{0}} \otimes_{R} x_{i_{2}} \cdots \otimes_{R} x_{i_{n}}\right)= \\
=\phi & \left(\sum_{k=0}^{n+1}(-1)^{k} \phi\left(x_{i_{0}} \otimes_{R} x_{i_{1}} \cdots x_{i_{k-1}} \otimes_{R}\left(\sum_{i=1}^{N} r_{i}(1)\right) \otimes_{R} x_{i_{k}} \cdots \otimes_{R} x_{i_{n}}\right)\right) \\
= & \sum_{k=0}^{n+1} r_{\eta(k)}^{i_{0}}\left(x_{i_{0}}\right) r_{\eta(k)}^{i_{1}}\left(x_{i_{1}}\right) \cdots\left(\sum_{i \in I / \zeta} r_{\eta(k)}^{i}(1)\right) \cdots r_{\eta(k)}^{i_{n}}\left(x_{i_{n}}\right) \\
= & \sum_{i \in I / \zeta} \sum_{k=0}^{n+1} r_{\eta(k)}^{i_{0}}\left(x_{i_{0}}\right) r_{\eta(k)}^{i_{1}}\left(x_{i_{1}}\right) \cdots r_{\eta(k)}^{i_{n}}\left(x_{i_{n}}\right)
\end{aligned}
$$

which ends the proof.

Remark 2. The trivial example of the functor $\mathcal{R}$ is given by a constant functor associating ring $R$ to every $\zeta \in P(\mathcal{I})$. The coring $\mathcal{C}(\mathcal{R})$ is then a full-matrix coring.

The homology of the associated Čech cochain complex is:

$$
H^{0}(S)=R, \quad H^{1}(S)=0
$$

Remark 3. If $X$ is a ringed space with the functor $\Psi$ from open subsets of $X$ to the category of unital rings, and $U_{i}, i \in \mathcal{I}$ is a finite covering of $X$, then we construct $\mathcal{R}$ by setting:

$$
\mathcal{R}\left(\left\{i_{1}, i_{2}, \ldots, i_{k}\right\}\right)=\Psi\left(U_{i_{1}} \cap U_{i_{2}} \cdots \cap U_{i_{k}}\right) .
$$

In the next section we shall discuss the relation of the constructed complex with the standard Cech cohomology.

\section{3 Čech cohomology of ringed algebras}

One of the examples of the functor $\mathcal{R}$ for a finite set $\mathcal{I}$ was based on the structure of a ringed space and a finite covering by open sets. We adapt the definition to the algebraic case:

Definition 2.5. We call the algebra $A$ ringed if there exists a functor $\Phi$ which associates to any ideal $J \subset A$ a ring $\Phi(J)$, and a ring morphism 
$\Phi_{J}: A / J \rightarrow R(J)$, such that if $J_{1} \subset J_{2}$ then the following diagram is commutative:

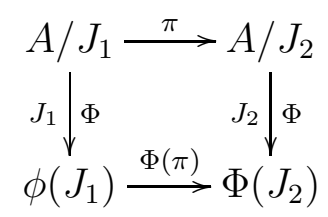

Remark 4. An example of the ringed algebra is given by taking $\Phi(J)=A / J$ and $\Phi_{J}$ the identity map.

Proposition 2.6. Assume now that $A$ is a ringed algebra with the ringed structure $\Phi$ and $\left\{J_{i}\right\}_{i \in \mathcal{I}}$ is a finite complete covering of $A$. Then by setting for every ordered subset of $\mathcal{I}, \zeta=\left\{i_{1}, \ldots, i_{k}\right\} \in P(\mathcal{I})$

$$
\mathcal{R}(\zeta)=\Phi\left(I_{i_{1}}+\cdots+I_{i_{k}}\right),
$$

and for the respective homomorphisms $r_{\zeta}^{\eta}: R(\eta) \rightarrow R(\zeta)$ to be images under $\Phi$ of projection morphisms. we obtain a functor $\mathcal{R}(\mathcal{I})$.

Definition 2.7. If $(A, \Phi)$ is a ringed algebra, and $\left\{I_{i}\right\}_{i \in \mathcal{I}}$ is its finite covering, we define the Cech cohomology of $A$ relative to the covering as the homology of the Cech cochain complex of the covering coring.

\section{Examples and applications}

Let $X$ be a topological space, and $\mathcal{O}_{X}$ be a presheaf of rings on $X$. We denote the functor associating a ring to any open set $U$ by $\Phi(U)$, and the ring morphisms corresponding to set inclusion $i_{U, V}: U \hookrightarrow V$ by $\Phi_{U}^{V}: \Phi(V) \rightarrow$ $\Phi(U)$.

Let $U_{i}, i \in I$ be a finite covering of $X$. Then from the presheaf $\mathcal{O}_{X}$ we can construct the data of a ringed space as in the previous section, the coring, the map $\phi$ and the respective cochain complexes. We have:

Lemma 3.1. The homology of the cochain complex $S(\mathcal{R})$ for the coring given by the data $I, \mathcal{O}_{X}$, is the Cech cohomology of $X$ relative to the covering $\left\{U_{i}\right\}_{I}$.

Proof. By rewriting the definition of $S^{n}, d^{\prime}$ using the sets $U_{i}$ and the intersections, we explicitly recover the definition of Cech homology. 
Remark 5. The construction of Čech cohomology is possible for any matrixtype coring, by using the canonical functor ringed structure $\Phi$ and the covering implicitly defined by the coring construction.

\section{Conclusions}

The formulation of noncommutative coverings in the language of algebra extensions and associated corings has enabled us to extend the definition of Cech cohomology to this setting.

The Čech cohomology is mostly of combinatorial nature and strongly depends on the properties of the underlying noncommutative covering. It would be interesting to know, whether for some types of noncommutative coverings one could establish relation between this cohomology and some other cohomology theory of the algebra.

\section{References}

[1] D. Calow, R. Matthes, Covering and gluing of algebras and differential algebras. J. Geom. Phys. 32 (2000), no. 4, 364-396.

[2] T, Brzeziński, R.Wisbauer, Corings and comodules. London Mathematical Society Lecture Note Series, 309. Cambridge University Press, Cambridge, 2003

[3] T. Brzeziński, A.P.Wrightson, Complete coverings and Galois corings. Int. J. Geom. Methods Mod. Phys. 2 (2005), no. 5, 751-757

[4] Bott, R., Tu, L. W.: Differential forms in algebraic topology. SpringerVerlag, Berlin, 1982 